\title{
Lattice points in large convex bodies, II
}

\author{
by
}

\author{
Ekkehard Krätzel* (Jena) and Werner Georg NowaK** (Wien)
}

1. Introduction. Let $\mathcal{B}$ denote a compact convex subset of $\mathbb{R}^{s}(s \geq 3)$ which contains the origin as an inner point. Suppose that $\mathcal{B}$ has a smooth boundary $\partial \mathcal{B}$ with finite nonzero Gaussian curvature throughout, and assume that the "canonical map" which sends every point $\mathbf{u}$ of the unit sphere in $\mathbb{R}^{s}$ to that point $\mathbf{x}$ of $\partial \mathcal{B}$ where the outward normal has the same direction, is one-one and of class $C^{\infty}$. We consider the number $A(t)$ of lattice points (of the standard lattice $\mathbb{Z}^{s}$ ) in the "blown-up" body $\sqrt{t} \mathcal{B}=\left\{\mathbf{x} \in \mathbb{R}^{s}\right.$ : $(1 / \sqrt{t}) \mathbf{x} \in \mathcal{B}\}$, where $t$ is a large real variable. As usual, we define the "lattice rest" by $P(t)=A(t)-V t^{s / 2}$, where $V$ is the volume of $\mathcal{B}$.

For such a general convex body $\mathcal{B}$, not too much progress has been made in our knowledge of this lattice point problem. A few years ago, the sharpest results were those of Hlawka [4] from 1950: Hlawka proved that

$$
P(t)=O\left(t^{s(s-1) / 2(s+1)}\right),
$$

and

$$
P(t)=\Omega\left(t^{(s-1) / 4}\right) .
$$

In recent times, the $\Omega$-estimate has been improved by the second-named author (see [12]-[14]). For every dimension $s \geq 4$, it is known that

$$
P(t)=\Omega_{ \pm}\left(t^{(s-1) / 4}(\log t)^{1 / 2-1 /(2 s)}\right) .
$$

(For $s=2,3$, one has a corresponding one-sided result.)

For the upper bound (1.1), the authors quite recently were able to obtain an improvement, for every $s \geq 3$ (see [10]). The result reads $P(t) \ll t^{\lambda^{*}(s)}$

* This article was prepared while the first-named author was visiting professor at Vienna University, in spring semester 1991.

** This paper is part of a research project supported by the Austrian "Fonds zur Förderung der wissenschaftlichen Forschung" (Nr. P8488-PHY). 
with

$$
\lambda^{*}(s) \stackrel{\text { def }}{=} \begin{cases}\frac{s}{2}-1+\frac{6}{7 s+4} & \text { for } 3 \leq s \leq 7, \\ \frac{s}{2}-1+\frac{5}{6 s+2} & \text { for } s \geq 8 .\end{cases}
$$

This was obtained by a combination of Hlawka's own method along with a classical estimate for one-dimensional exponential sums.

In the present article, we establish an upper bound for $P(t)$ which is still sharper than this last result, for every dimension $s \geq 3$.

Theorem. For the lattice rest $P(t)$ of the convex body $\sqrt{t} \mathcal{B}$ in $\mathbb{R}^{s}(s \geq 3)$, where $\mathcal{B}$ satisfies the conditions stated above, we have

$$
P(t) \ll \begin{cases}t^{s / 2-1+4 /(5 s+2)}(\log t)^{10 /(5 s+2)} & \text { for } 3 \leq s \leq 6, \\ t^{s / 2-1+3 /(4 s)}(\log t)^{2 / s} & \text { for } s \geq 7 .\end{cases}
$$

(Throughout the paper, all constants implied in the symbols $O, \ll$ and $\asymp$ depend only on the convex body $\mathcal{B}$, unless explicitly stated otherwise.)

R e mark. For planar domains, the problem has been attacked by Huxley [6], [7] who applied his refined variant of the "Discrete Hardy-Littlewood Method" which originally is due to Bombieri, Iwaniec and Mozzochi. He obtained the result

$$
P(t)=O\left(t^{7 / 22}(\log t)^{47 / 22}\right),
$$

for every planar domain $\mathcal{B}$ satisfying the conditions stated above. Quite recently (at an Oberwolfach meeting in March 1991), Huxley has announced a further improvement of his analysis which allows him to reduce the exponent $\frac{7}{22}$ to $\frac{23}{73}+\varepsilon$.

Up to the moment, no one has found a genuine extension of the "Discrete Hardy-Littlewood Method" to dimensions $s \geq 3$. Our method of proof will thus be based on classical tools, starting with Landau's averaging technique. By Hlawka's expansions of exponential integrals over such general convex bodies, one arrives at certain $s$-fold exponential sums. We estimate these by Titchmarsh's two-dimensional method ([15], [16]) (which has been refined and elaborated in the first-named author's textbook [9]), preceded by a suitable number of "Weyl's steps".

2. Preliminaries. As in part I of this work [10], we recall a few facts and notations which are essential for the Landau technique.

For every function $F$ integrable on every compact subinterval of $\mathbb{R}_{0}^{+}$, and every $r \in \mathbb{N}$, we denote by $F_{(r)}$ the $r$-fold anti-derivative

$$
F_{(r)}(t) \stackrel{\text { def }}{=} \frac{1}{(r-1) !} \int_{0}^{t}(t-w)^{r-1} F(w) d w \quad(w \geq 0) .
$$


For $r$ sufficiently large, e.g. $r=[(s+1) / 2]$, it follows by Poisson's formula (cf. Bochner [1], or Hlawka [4]) that

$$
A_{(r)}(t)=\frac{1}{r !} \sum_{\mathbf{n} \in \sqrt{t} \mathcal{B}, \mathbf{n} \in \mathbb{Z}^{s}}\left(t-\varphi^{2}(\mathbf{n})\right)^{r}=\frac{\Gamma(s / 2+1)}{\Gamma(s / 2+r)} V t^{s / 2+r}+P_{(r)}(t),
$$

where $\varphi$ is the distance function of the convex body $\mathcal{B}$,

$$
\begin{gathered}
P_{(r)}(t)=\sum_{\mathbf{m} \in \mathbb{Z}^{s}, \mathbf{m} \neq \mathbf{0}} I_{r}(\mathbf{m}, t), \\
I_{p}(\mathbf{m}, t) \stackrel{\text { def }}{=} \frac{1}{p !} \int_{\varphi^{2}(\mathbf{u}) \leq t}\left(t-\varphi^{2}(\mathbf{u})\right)^{p} e(\mathbf{m u}) d \mathbf{u}
\end{gathered}
$$

for each $p \in \mathbb{N}_{0}, e(w) \stackrel{\text { def }}{=} e^{2 \pi i w}$. We recall that

$$
\frac{d}{d t}\left(I_{p+1}(\mathbf{m}, t)\right)=I_{p}(\mathbf{m}, t) .
$$

Furthermore, we denote by $\Delta_{z}^{(r)}$ the $r$-fold iterated difference operator, i.e.

$$
\Delta_{z}^{(r)}(F(t)) \stackrel{\text { def }}{=} \sum_{j=0}^{r}(-1)^{r-j}\left(\begin{array}{l}
r \\
j
\end{array}\right) F(t+j z)
$$

for positive real numbers $t$ and $z$. Then

$$
\Delta_{z}^{(r)}\left(F_{(r)}(t)\right)=\int_{t}^{t+z} d w_{1} \int_{w_{1}}^{w_{1}+z} d w_{2} \ldots \int_{w_{r-1}}^{w_{r-1}+z} F\left(w_{r}\right) d w_{r}
$$

for every function $F$ which is piecewise continuous and integrable on each compact subinterval of $\mathbb{R}_{0}^{+}$.

Our next important tool is Hlawka's asymptotic expansion for the integrals $I_{p}(\mathbf{m}, t)$ :

Lemma 1. For $\mathbf{m} \in \mathbb{Z}^{s}, \mathbf{m} \neq \mathbf{0}, p \in \mathbb{N}_{0}$, and a large real parameter $w$,

$$
\begin{aligned}
& I_{p}(\mathbf{m}, w)=\left(\gamma_{p}(\mathbf{m}) e\left(H(\mathbf{m}) \sqrt{w}-\tau_{p}\right)\right. \\
& \left.\quad+\gamma_{p}(-\mathbf{m}) e\left(-H(-\mathbf{m}) \sqrt{w}+\tau_{p}\right)\right) w^{\frac{1}{2}\left(\frac{s-1}{2}+p\right)}\left(1+O\left(\|\mathbf{m}\|^{-1} w^{-1 / 2}\right)\right),
\end{aligned}
$$

where $\tau_{p}=\frac{1}{4}\left(p+\frac{s+1}{2}\right), H$ is the tac-function of the convex body $\mathcal{B}$ ( "Stützfunktion", cf. Bonnesen and Fenchel [2]), thus a positive $C^{\infty}$-function on $\mathbb{R}^{s}-\{\mathbf{0}\}$ which is homogeneous of degree 1 , and $\gamma_{p}$ is a certain positive $C^{\infty}$-function on $\mathbb{R}^{s}-\{\mathbf{0}\}$ (given explicitly by Hlawka [4]), homogeneous of degree

$$
-d_{p} \stackrel{\text { def }}{=}-p-\frac{s+1}{2}
$$

Pr o of. This is Hlawka's Satz 2 in [5]. (See also Satz 5 in Hlawka [4].) 
LEMma 2. Let $\mathcal{D}$ be a polygon in $\mathbb{R}^{2}$ with $O(1)$ vertices which is contained in a two-dimensional interval $I=I_{1} \times I_{2}$ with side lengths $L\left(I_{1}\right), L\left(I_{2}\right)$. Let $k \geq 0$ be a fixed integer and let $f(\alpha, \beta)$ denote a function of two real variables with continuous partial derivatives up to order $k+3$ on

$$
I^{*}=\left\{\left(u+\theta k L\left(I_{1}\right), v\right) \in \mathbb{R}^{2}: u \in I_{1}, 0 \leq \theta \leq 1, v \in I_{2}\right\} .
$$

Suppose that $\Lambda$ and $M$ are real parameters $\geq 1$ with $L\left(I_{1}\right), L\left(I_{2}\right) \ll M$, such that the order relations

$$
\begin{gathered}
\left|\frac{\partial^{j} f}{\partial \alpha^{j}}\right| \asymp \Lambda M^{1-j} \quad(j=1, \ldots, k+2), \\
\left|\frac{\partial^{k+2} f}{\partial \alpha^{k} \partial \beta^{2}}\right| \asymp \Lambda M^{-k-1}, \quad\left|\frac{\partial^{k+2} f}{\partial \alpha^{k+1} \partial \beta}\right| \asymp \Lambda M^{-k-1},
\end{gathered}
$$

and

$$
\left|\frac{\partial^{k+2} f}{\partial \alpha^{k+2}} \frac{\partial^{k+2} f}{\partial \alpha^{k} \partial \beta^{2}}-\left(\frac{\partial^{k+2} f}{\partial \alpha^{k+1} \partial \beta}\right)^{2}\right| \gg \Lambda^{2} M^{-2 k-2}
$$

are satisfied on $I^{*}$ throughout. Suppose further that each partial derivative of $f$ of order $j \leq k+3$ is $\ll \Lambda M^{1-j}$ on $I^{*}$. Then

$$
\sum_{\left(n_{1}, n_{2}\right) \in \mathcal{D} \cap \mathbb{Z}^{2}} e\left(f\left(n_{1}, n_{2}\right)\right) \ll \Lambda^{\omega_{k}} M^{2-(k+1) \omega_{k}}(1+\log \Lambda+\log M),
$$

where $\omega_{k}=1 /\left(2^{k} 3-2\right)$ for short and the constant implied in this estimate depends only on the various constants implied in the order symbols of the suppositions.

Pr o of. This is a special case of Theorem 2.22 in Krätzel [9], p. 84, with a suitable change of notation.

We now establish a pivotal auxiliary result which will enable us to apply this deep exponential sum estimate in our general situation.

Lemma 3. Let $H(\mathbf{u})$ denote the tac-function of a convex body $\mathcal{B}$ in $\mathbb{R}^{s}$, where $s \geq 3$, which possesses the properties stated in the introduction. For nonzero vectors $\mathbf{p}, \mathbf{v}, \mathbf{w} \in \mathbb{R}^{s}$, define a function $F$ of two real variables by

$$
F(\alpha, \beta)=F(\alpha, \beta ; \mathbf{p}, \mathbf{v}, \mathbf{w})=H(\mathbf{p}+\alpha \mathbf{v}+\beta \mathbf{w}) .
$$

Let $k \geq 0$ be an arbitrary fixed integer. Then for any given $\mathbf{p} \in \mathbb{R}^{s}, \mathbf{p} \neq \mathbf{0}$, there exist vectors $\mathbf{v}, \mathbf{w} \in \mathbb{R}^{s}$ such that none of the equalities

$$
\begin{aligned}
&\left.\frac{\partial^{j} F}{\partial \alpha^{j}}\right|_{(\alpha, \beta)=(0,0)}=0 \quad(j=1,2, \ldots, k+2), \\
&\left.\frac{\partial^{k+2} F}{\partial \alpha^{k} \partial \beta^{2}}\right|_{(\alpha, \beta)=(0,0)}=0,\left.\quad \frac{\partial^{k+2} F}{\partial \alpha^{k+1} \partial \beta}\right|_{(\alpha, \beta)=(0,0)}=0,
\end{aligned}
$$


and

$$
\begin{aligned}
h_{k} & =h_{k}(\mathbf{p} ; \mathbf{v}, \mathbf{w}) \\
& \left.\stackrel{\text { def }}{=}\left(\frac{\partial^{k+2} F}{\partial \alpha^{k+2}} \frac{\partial^{k+2} F}{\partial \alpha^{k} \partial \beta^{2}}-\left(\frac{\partial^{k+2} F}{\partial \alpha^{k+1} \partial \beta}\right)^{2}\right)\right|_{(\alpha, \beta)=(0,0)}=0
\end{aligned}
$$

is satisfied.

Proof. We first consider $h_{k}$ which involves the most difficulties. By a straightforward calculation,

$$
h_{k}=\sum_{\substack{1 \leq a_{1} \leq \ldots \leq a_{2 k+2} \leq s \\ 1 \leq b_{1} \leq b_{2} \leq s}} C\left(a_{1}, \ldots, a_{2 k+2} ; b_{1}, b_{2}\right) v_{a_{1}} \ldots v_{a_{2 k+2}} w_{b_{1}} w_{b_{2}}
$$

where

$$
\begin{aligned}
& C\left(a_{1}, \ldots, a_{2 k+2} ; b_{1}, b_{2}\right) \\
& =\sum_{\text {(A) }} \sum_{\text {(B) }} \operatorname{det}\left(\begin{array}{cc}
H_{a_{1}^{\prime} a_{2}^{\prime} a_{3}^{\prime} \ldots a_{k+2}^{\prime}} & H_{a_{1}^{\prime \prime} b_{2}^{\prime \prime} a_{k+3}^{\prime \prime} \ldots a_{2 k+2}^{\prime \prime}} \\
H_{b_{1}^{\prime \prime} a_{2}^{\prime \prime} a_{3}^{\prime \prime} \ldots a_{k+2}^{\prime \prime}} & H_{b_{1}^{\prime} b_{2}^{\prime} a_{k+3}^{\prime} \ldots a_{2 k+2}^{\prime}}^{\prime}
\end{array}\right)(\mathbf{p}),
\end{aligned}
$$

where the summation conditions (A) and (B) are defined (throughout the sequel) by

(A): $\left(a_{1}^{\prime}, \ldots, a_{2 k+2}^{\prime}\right) \in P\left(a_{1}, \ldots, a_{2 k+2}\right),\left(a_{1}^{\prime \prime}, \ldots, a_{2 k+2}^{\prime \prime}\right) \in P\left(a_{1}, \ldots, a_{2 k+2}\right)$, (B): $\left(b_{1}^{\prime}, b_{2}^{\prime}\right) \in P\left(b_{1}, b_{2}\right),\left(b_{1}^{\prime \prime}, b_{2}^{\prime \prime}\right) \in P\left(b_{1}, b_{2}\right)$,

and $P(\cdot)$ denotes the set of all permutations with repetitions of the elements given in the bracket; the subscripts of $H$ denote iterated partial derivatives as usual. We now show, by induction on $k \geq 0$, that for every $\mathbf{p} \in \mathbb{R}^{s}$, $\mathbf{p} \neq \mathbf{0}$, there exists at least one nonzero coefficient $C(\cdot)$ in $(2.12)$.

To show this for $k=0$, we recall what is known about the matrix $T=\left(H_{i j}(\mathbf{p})\right)$ where $H_{i j}$ are all partial derivatives of order 2 . According to Bonnesen-Fenchel [2], p. $61 \mathrm{ff}$., the rank of this matrix is equal to $s-1$, and its nonzero eigenvalues are all positive. Thus $\left(^{1}\right)$ there exist $a, b \in\{1, \ldots, s\}$ such that

$$
C(a, a ; b, b)=\operatorname{det}\left(\begin{array}{ll}
H_{a a} & H_{a b} \\
H_{b a} & H_{b b}
\end{array}\right)(\mathbf{p})>0 .
$$

Suppose now the assertion is true for some $k \geq 0$ : Let

$$
C\left(a_{1}, \ldots, a_{2 k+2} ; b_{1}, b_{2}\right) \neq 0 .
$$

$\left({ }^{1}\right)$ This conclusion is an elementary exercise in linear algebra which we leave to the reader. 
For real variables $\xi, \eta>0$, let us consider the function

$$
\varphi(\xi, \eta) \stackrel{\text { def }}{=} \sum_{(\mathrm{A})} \sum_{(\mathrm{B})} \operatorname{det}\left(\begin{array}{cc}
H_{a_{1}^{\prime} a_{2}^{\prime} a_{3}^{\prime} \ldots a_{k+2}^{\prime}}(\xi \mathbf{p}) & H_{a_{1}^{\prime \prime} b_{2}^{\prime \prime} a_{k+3}^{\prime \prime} \ldots a_{2 k+2}^{\prime \prime}}(\eta \mathbf{p}) \\
H_{b_{1}^{\prime \prime} a_{2}^{\prime \prime} a_{3}^{\prime \prime} \ldots a_{k+2}^{\prime \prime}}(\xi \mathbf{p}) & H_{b_{1}^{\prime} b_{2}^{\prime} a_{k+3}^{\prime} \ldots a_{2 k+2}^{\prime}}(\eta \mathbf{p})
\end{array}\right) .
$$

By homogeneity of $H$ and its partial derivatives, this is equal to

$$
(\xi \eta)^{-k-1} C\left(a_{1}, \ldots, a_{2 k+2} ; b_{1}, b_{2}\right)
$$

hence

$$
\left.\frac{\partial^{2} \varphi}{\partial \xi \partial \eta}\right|_{(\xi, \eta)=(1,1)} \neq 0
$$

On the other hand, by a direct computation,

$$
\begin{aligned}
&\left.\frac{\partial^{2} \varphi}{\partial \xi \partial \eta}\right|_{(\xi, \eta)=(1,1)}=\sum_{1 \leq c_{1} \leq c_{2} \leq s} p_{c_{1}} p_{c_{2}} \\
& \times \sum_{(\mathrm{A})} \sum_{(\mathrm{B})} \sum_{\substack{\left(c_{1}^{\prime}, c_{2}^{\prime}\right) \in P\left(c_{1}, c_{2}\right) \\
\left(c_{1}^{\prime \prime}, c_{2}^{\prime \prime}\right) \in P\left(c_{1}, c_{2}\right)}} \operatorname{det}\left(\begin{array}{cc}
H_{c_{1}^{\prime} a_{1}^{\prime} a_{2}^{\prime} a_{3}^{\prime} \ldots a_{k+2}^{\prime}} & H_{c_{1}^{\prime \prime} a_{1}^{\prime \prime} b_{2}^{\prime \prime} a_{k+3}^{\prime \prime} \ldots a_{2 k+2}^{\prime \prime}} \\
H_{c_{2}^{\prime \prime} b_{1}^{\prime \prime} a_{2}^{\prime \prime} a_{3}^{\prime \prime} \ldots a_{k+2}^{\prime \prime}} & H_{c_{2}^{\prime} b_{1}^{\prime} b_{2}^{\prime} a_{k+3}^{\prime} \ldots a_{2 k+2}^{\prime}}^{\prime}
\end{array}\right)(\mathbf{p}) .
\end{aligned}
$$

Thus it is impossible that all of the inner sums here vanish. Renaming the subscripts, we immediately infer the existence of a nonzero coefficient $C(\cdot)$ in (2.12) for $k+1$.

Moreover, by a well-known formula,

$$
\begin{array}{r}
\left.\frac{\partial^{a+b} F}{\partial \alpha^{a} \partial \beta^{b}}\right|_{(\alpha, \beta)=(0,0)}=\sum_{\begin{array}{c}
e_{1}+\ldots+e_{s}=a \\
f_{1}+\ldots+f_{s}=b
\end{array}} \frac{a !}{e_{1} ! \ldots e_{s} !} \frac{b !}{f_{1} ! \ldots f_{s} !} \frac{\partial^{a+b} H}{\partial u_{1}^{e_{1}+f_{1}} \ldots \partial u_{s}^{e_{s}+f_{s}}}(\mathbf{p}) \\
\times v_{1}^{e_{1}} \ldots v_{s}^{e_{s}} w_{1}^{f_{1}} \ldots w_{s}^{f_{s}},
\end{array}
$$

where $a, b$ and $e_{j}, f_{j}$ are in $\mathbb{N}_{0}$. It is impossible that all partial derivatives of $H$ of order $a+b \geq 1$ vanish at some (arbitrary, but fixed) point $\mathbf{p} \in \mathbb{R}^{s}$. (For $a+b \geq 2$, this follows from the above argument. For $a+b=1$, it suffices to observe that $\frac{d}{d \xi} H(\xi \mathbf{p})=H(\mathbf{p}) \neq 0$ for $\xi>0$, since $H$ is positive and homogeneous of degree 1.) Consequently, each one of the equations (2.9) to (2.11) defines a set of $2 s$-tuples $\left(v_{1}, \ldots, v_{s}, w_{1}, \ldots, w_{s}\right)$ of Lebesgue measure zero in $\mathbb{R}^{2 s}$. (Note that the left-hand side is always a nontrivial homogeneous polynomial.) From this the assertion of Lemma 3 is immediate.

LEMMA 4. Let $\mathcal{D}$ be a subset of $\mathbb{R}^{2}$ which is contained in a compact two-dimensional interval $I$ whose side lengths are $\ll M$. Let $\gamma$ denote a function of two real variables on I with continuous partial derivatives up to order 2. Suppose that

$$
\gamma \ll G, \quad \gamma_{1}, \gamma_{2} \ll G / M, \quad \gamma_{12} \ll G / M^{2}
$$


on all of $I$. (Here $M \geq 1$ and $G$ are real parameters, and the subscripts denote partial derivatives.) Furthermore, let $\eta$ denote a function defined on $\mathcal{D} \cap \mathbb{Z}^{2}$. Then

$$
\sum_{\left(n_{1}, n_{2}\right) \in \mathcal{D} \cap \mathbb{Z}^{2}} \gamma\left(n_{1}, n_{2}\right) \eta\left(n_{1}, n_{2}\right) \ll G \max _{(u, v) \in I} \sum_{\substack{\left(n_{1}, n_{2}\right) \in \mathcal{D} \cap \mathbb{Z}^{2} \\ n_{1} \leq u, n_{2} \leq v}} \eta\left(n_{1}, n_{2}\right),
$$

where the constant implied in this estimate depends only on the constants implied in the order symbols of the suppositions.

Proof. This result on partial summation in two dimensions can be easily derived from formula (1.5) on p. 19 of [9], or also be considered as a special case of Theorem 1.6 in the same book, p. 24. One only has to put $\eta=0$ on $(I-\mathcal{D}) \cap \mathbb{Z}^{2}$.

3. Proof of the Theorem. The first step is quite the same as in [10]. Thus $t$ is a large real variable and $z=z(t)$ is a certain (continuous) function of $t$ to be fixed later on (see (3.6) below), such that $z=o(t)$ as $t \rightarrow \infty$. It is an immediate consequence of $(2.1)$ and (2.6) that, for $r \stackrel{\text { def }}{=}[(s+1) / 2]$,

$$
\begin{aligned}
A(t) & \leq z^{-r} \Delta_{z}^{(r)}\left(A_{(r)}(t)\right) \\
& =z^{-r} V \frac{\Gamma(s / 2+1)}{\Gamma(s / 2+r)} \Delta_{z}^{(r)}\left(t^{s / 2+r}\right)+z^{-r} \Delta_{z}^{(r)}\left(P_{(r)}(t)\right) \\
& =V t^{s / 2}+O\left(t^{s / 2-1} z\right)+z^{-r} \Delta_{z}^{(r)}\left(P_{(r)}(t)\right) .
\end{aligned}
$$

On the other hand, with $t^{*}=t+r z$, we have

$$
A\left(t^{*}\right) \geq z^{-r} \Delta_{z}^{(r)}\left(A_{(r)}(t)\right),
$$

and

$$
t^{*} \asymp t, \quad\left(t^{*}\right)^{s / 2}=t^{s / 2}+O\left(t^{s / 2-1} z\right) .
$$

Hence it will suffice to estimate the right-hand side of (3.1).

Let

$$
y=\sqrt{t} / z .
$$

(It will be clear from (3.6) below that $y \rightarrow \infty$ with $t$.) Recalling (2.2), we obtain

$$
\begin{aligned}
\Delta_{z}^{(r)}\left(P_{(r)}(t)\right) & =\sum_{\mathbf{m} \in \mathbb{Z}^{s}, \mathbf{m} \neq \mathbf{0}} \Delta_{z}^{(r)}\left(I_{r}(\mathbf{m}, t)\right) \\
& =\sum_{0<\|\mathbf{m}\|_{\infty} \leq y}+\sum_{\|\mathbf{m}\|_{\infty}>y} .
\end{aligned}
$$

(Here and throughout the sequel, $\|\cdot\|_{\infty}$ denotes the maximum norm in $\mathbb{R}^{s}$.) Using (2.6), (2.4), and the linearity of the $\Delta$-operator for the first part of 
the sum, and (2.5) for the second part, we obtain (cf. [10] for a detailed calculation)

$$
\begin{aligned}
& \Delta_{z}^{(r)}\left(P_{(r)}(t)\right) \\
\ll & z^{r} \max _{t \leq w \leq t+r z}\left|\sum_{0<\|\mathbf{m}\|_{\infty} \leq y} I_{0}(\mathbf{m}, w)\right|+\max _{t \leq w \leq t+r z}\left|\sum_{\|\mathbf{m}\|_{\infty}>y} I_{r}(\mathbf{m}, w)\right| .
\end{aligned}
$$

In what follows, $p$ is either 0 or $r, M \geq 1$ is a real variable, $k$ is either 1 or 2 (it depends on the dimension $s$ and will be fixed later). Furthermore, let

$$
\mathcal{K}_{0} \stackrel{\text { def }}{=}\left\{\mathbf{p} \in \mathbb{R}^{s}: 1<\|\mathbf{p}\|_{\infty} \leq 2\right\} .
$$

To estimate the sums on the right-hand side of (3.5), we split up their domains of summation and consider first only the main terms of the asymptotic expansion in Lemma 1. We thus obtain exponential sums

$$
\Sigma_{M} \stackrel{\text { def }}{=} \sum_{\mathbf{m} \in M \mathcal{K}_{0}} \gamma_{p}(\mathbf{m}) e(H(\mathbf{m}) \sqrt{w}) \quad(w \asymp t) .
$$

For nonzero vectors $\mathbf{p}, \mathbf{v}, \mathbf{w}$ in $\mathbb{R}^{s}$, let $F$ be defined by (2.8) and put, for $i, j \in \mathbb{N}_{0}$,

$$
\left.\delta_{i, j}(\mathbf{p} ; \mathbf{v}, \mathbf{w}) \stackrel{\text { def }}{=} \frac{\partial^{i+j} F}{\partial \alpha^{i} \partial \beta^{j}}\right|_{(\alpha, \beta)=(0,0)},
$$

and

$$
\begin{aligned}
& \mu_{F}(\mathbf{p} ; \mathbf{v}, \mathbf{w}) \stackrel{\text { def }}{=} \\
& \inf \left\{\inf _{1 \leq i \leq k+2}\left|\delta_{i, 0}(\mathbf{p} ; \mathbf{v}, \mathbf{w})\right|,\left|\delta_{k+1,1}(\mathbf{p} ; \mathbf{v}, \mathbf{w})\right|,\left|\delta_{k, 2}(\mathbf{p} ; \mathbf{v}, \mathbf{w})\right|,\left|h_{k}(\mathbf{p} ; \mathbf{v}, \mathbf{w})\right|\right\},
\end{aligned}
$$

where $h_{k}$ is defined in (2.11).

We now apply Lemma 3, along with an obvious continuity argument. For every point $\mathbf{p} \in \overline{\mathcal{K}}_{0}$ (the bar denotes the topological closure) there exist rational vectors $\mathbf{v}^{*}=\mathbf{v}^{*}(\mathbf{p}) \neq \mathbf{0}$ and $\mathbf{w}^{*}=\mathbf{w}^{*}(\mathbf{p}) \neq \mathbf{0}$ (not collinear), and a positive number $\varepsilon(\mathbf{p})$ such that

$$
\mu_{F}\left(\mathbf{p}^{\prime} ; \mathbf{v}^{*}, \mathbf{w}^{*}\right)>0 \quad \text { for all } \mathbf{p}^{\prime} \in \mathbb{R}^{s} \text { with }\left\|\mathbf{p}^{\prime}-\mathbf{p}\right\|_{\infty}<5 \sqrt{s} \varepsilon(\mathbf{p}) .
$$

Put

$$
\mathcal{K}^{*}(\mathbf{p}) \stackrel{\text { def }}{=}\left\{\mathbf{p}^{\prime} \in \mathbb{R}^{s}:\left\|\mathbf{p}^{\prime}-\mathbf{p}\right\|_{\infty}<\varepsilon(\mathbf{p})\right\} .
$$

Then $\overline{\mathcal{K}}_{0}$ is covered by the union of all open cubes $\mathcal{K}^{*}(\mathbf{p})$ with $\mathbf{p} \in \overline{\mathcal{K}}_{0}$, and, by compactness, even by finitely many of them, say

$$
\overline{\mathcal{K}}_{0} \subset \bigcup_{j=1}^{J} \mathcal{K}^{*}\left(\mathbf{p}^{(j)}\right)
$$


We now define sets $\mathcal{K}_{j} \subseteq \mathcal{K}^{*}\left(\mathbf{p}^{(j)}\right), j=1, \ldots, J$, by

$$
\mathcal{K}_{j} \stackrel{\text { def }}{=} \mathcal{K}_{0} \cap\left(\mathcal{K}^{*}\left(\mathbf{p}^{(j)}\right)-\bigcup_{1 \leq i<j} \mathcal{K}^{*}\left(\mathbf{p}^{(i)}\right)\right) .
$$

These sets are all disjoint, their union is $\mathcal{K}_{0}$, and they have the property that the intersection of any (two-dimensional) plane with any $\mathcal{K}_{j}$ gives at most $O(1)$ polygons with $O(1)$ vertices. For each $j=1, \ldots, J$, we can find integer vectors $\mathbf{v}^{(j)}, \mathbf{w}^{(j)}$ which are collinear to $\mathbf{v}^{*}\left(\mathbf{p}^{(j)}\right), \mathbf{w}^{*}\left(\mathbf{p}^{(j)}\right)$, respectively, such that the greatest common divisor of the components is equal to 1 for either of them.

We decompose the above exponential sum:

$$
\Sigma_{M}=\sum_{j=1}^{J} \sum_{\mathbf{m} \in M \mathcal{K}_{j}} \gamma_{p}(\mathbf{m}) e(\sqrt{w} H(\mathbf{m})) .
$$

For each fixed $j=1, \ldots, J$, there exist (integer) vectors $\mathbf{v}^{(j, 2)}, \ldots, \mathbf{v}^{(j, s)}$ which together with $\mathbf{v}^{(j)}$ form a basis of $\mathbb{Z}^{s}$, such that

$$
\mathbf{w}^{(j)}=c_{1} \mathbf{v}^{(j)}+c_{2} \mathbf{v}^{(j, 2)}
$$

with integers $0 \leq c_{1}<c_{2}$ (cf. Lekkerkerker [11], p. 20). Every $\mathbf{m} \in M \mathcal{K}_{j}$ has a unique representation

$$
\begin{aligned}
\mathbf{m} & =n_{1}^{\prime} \mathbf{v}^{(j)}+n_{2}^{\prime} \mathbf{v}^{(j, 2)}+\ldots+n_{s}^{\prime} \mathbf{v}^{(j, s)} \\
& =\left(n_{1}^{\prime}-\frac{c_{1}}{c_{2}} n_{2}^{\prime}\right) \mathbf{v}^{(j)}+\frac{1}{c_{2}} n_{2}^{\prime} \mathbf{w}^{(j)}+\sum_{i=3}^{s} n_{i}^{\prime} \mathbf{v}^{(j, i)},
\end{aligned}
$$

with $n_{i}^{\prime} \in \mathbb{Z}$. Consider those $\mathbf{m}$ for which $n_{2}^{\prime}$ lies in a fixed residue class $\varrho$ modulo $c_{2}\left(0 \leq \varrho<c_{2}\right.$; note that $\left.c_{2}=O(1)\right)$, and put $n_{2}^{\prime}=c_{2} n_{2}+\varrho$. Then we get

$$
\begin{gathered}
\mathbf{m}=n_{1} \mathbf{v}^{(j)}+n_{2} \mathbf{w}^{(j)}+\mathbf{b}^{(j)} \\
\mathbf{b}^{(j)}=\mathbf{b}^{(j)}\left(\varrho ; n_{3}, \ldots, n_{s}\right)=-\frac{c_{1}}{c_{2}} \varrho \mathbf{v}^{(j)}+\frac{1}{c_{2}} \varrho \mathbf{w}^{(j)}+\sum_{i=3}^{s} n_{i} \mathbf{v}^{(j, i)}
\end{gathered}
$$

with $n_{1}=n_{1}^{\prime}-\left(c_{1} / c_{2}\right)\left(n_{2}^{\prime}-\varrho\right), n_{i}=n_{i}^{\prime}$ for $3 \leq i \leq s$. We keep $j$, $\varrho$, and $n_{3}, \ldots, n_{s}$ fixed and denote by $\mathcal{D}$ any of the $O(1)$ polygons which make up the range for $n_{1}, n_{2}$, corresponding to $\mathbf{m} \in M \mathcal{K}_{j}$. Put

$$
f(\alpha, \beta) \stackrel{\text { def }}{=} \sqrt{w} H\left(\alpha \mathbf{v}^{(j)}+\beta \mathbf{w}^{(j)}+\mathbf{b}^{(j)}\right) .
$$

Then it is clear by the homogeneity of $H$ and our construction that the conditions of Lemma 2 are satisfied with $\Lambda=\sqrt{t}$, as long as $\alpha \mathbf{v}^{(j)}+\beta \mathbf{w}^{(j)}+\mathbf{b}^{(j)}$ lies in $M \mathcal{K}_{j}$. If $\mathcal{D}^{\prime}$ denotes the intersection of $\mathcal{D}$ with any two-dimensional 
interval, we may thus infer from Lemma 2 that

$$
\sum_{\left(n_{1}, n_{2}\right) \in \mathcal{D}^{\prime} \cap \mathbb{Z}^{2}} e\left(f\left(n_{1}, n_{2}\right)\right) \ll t^{\omega_{k} / 2} M^{2-(k+1) \omega_{k}} \log (M t) .
$$

To deal with the factor $\gamma_{p}(\mathbf{m})$, we observe that every partial derivative of $\gamma_{p}(\cdot)$ of order $R \geq 0$ is homogeneous of degree $-d_{p}-R$ (recall (2.7)).

Hence $\gamma_{p}\left(\alpha \mathbf{v}^{(j)}+\beta \mathbf{w}^{(j)}+\mathbf{b}^{(j)}\right)$ (as a function of $(\alpha, \beta)$ ) satisfies the assumptions of Lemma 4 with $G \asymp M^{-d_{p}}$. This implies that

$$
\begin{gathered}
\sum_{\left(n_{1}, n_{2}\right) \in \mathcal{D} \cap \mathbb{Z}^{2}} \gamma_{p}\left(n_{1} \mathbf{v}^{(j)}+n_{2} \mathbf{w}^{(j)}+\mathbf{b}^{(j)}\right) e\left(\sqrt{w} H\left(n_{1} \mathbf{v}^{(j)}+n_{2} \mathbf{w}^{(j)}+\mathbf{b}^{(j)}\right)\right) \\
\ll t^{\omega_{k} / 2} M^{2-d_{p}-\omega_{k}(k+1)} \log (M t) .
\end{gathered}
$$

Summation over $n_{3}, \ldots, n_{s}$ leads to an extra factor $M^{s-2}$, since by construction the values of all of these variables are $\ll M$, as long as $\mathbf{m} \in M \mathcal{K}_{0}$. Furthermore, it now is trivial that the order term of Lemma 1 gives a contribution which is smaller than the bound we already have.

Finally, we let $M$ run through the sequence $y / 2, y / 4, \ldots$ for $p=0$, and through $y, 2 y, 4 y, \ldots$ for $p=r$. The exponent of $M$ is positive in the first case and negative in the second case, thus it follows from (3.5) that

$$
\begin{aligned}
z^{-r} \Delta_{z}^{(r)}\left(P_{(r)}(t)\right) & \ll t^{\omega_{k} / 2+(s-1) / 4} y^{(s-1) / 2-(k+1) \omega_{k}}\left(1+t^{s / 2} y^{-r} z^{-r}\right) \log t \\
& \ll t^{(s-1) / 2-k \omega_{k} / 2} z^{-(s-1) / 2+(k+1) \omega_{k}} \log t,
\end{aligned}
$$

in view of (3.3). Balancing the first order term here against the term $O\left(t^{s / 2-1} z\right)$ in (3.1), we find it optimal to choose

$$
z=t^{\left(1-k \omega_{k}\right) /\left(s+1-2 \omega_{k}(k+1)\right)}(\log t)^{2 /\left(s+1-2 \omega_{k}(k+1)\right)} .
$$

Inserting our estimate into (3.1), we thus obtain

$$
z^{-r} \Delta_{z}^{(r)}\left(A_{(r)}(t)\right)=V t^{s / 2}+O\left(t^{\lambda(s)}(\log t)^{\nu(s)}\right),
$$

with

$$
\lambda(s)=\frac{s}{2}-1+\frac{1-k \omega_{k}}{s+1-2 \omega_{k}(k+1)}, \quad \nu(s)=\frac{2}{s+1-2 \omega_{k}(k+1)} .
$$

We now choose $k=2$ for $3 \leq s \leq 6$, and $k=1$ for $s \geq 7$. This gives just the values of $\lambda(s)$ and $\nu(s)$ as stated in the Theorem.

By combining (3.7) with (3.1) and (3.2), our proof is complete.

\section{References}

[1] S. Bochner, Die Poissonsche Summationsformel in mehreren Veränderlichen, Math. Ann. 106 (1932), 56-63.

[2] T. Bonnesen und W. Fenchel, Theorie der konvexen Körper, Springer, Berlin 1934. 
[3] F. Fricker, Einführung in die Gitterpunktlehre, Birkhäuser, Basel 1982.

[4] E. Hlawka, Über Integrale auf konvexen Körpern I, Monatsh. Math. 54 (1950), $1-36$.

[5] -, Integrale auf konvexen Körpern II, ibid. 81-99.

[6] M. N. Huxley, Exponential sums and lattice points, Proc. London Math. Soc. (3) 60 (1990), 471-502.

[7] -, Area, lattice points and exponential sums, preprint, 1991.

[8] M. N. Huxley and N. Watt, Exponential sums and the Riemann zeta function, Proc. London Math. Soc. (3) 57 (1988), 1-24.

[9] E. Krätzel, Lattice Points, Kluwer, Dordrecht 1988.

[10] E. Krätzel and W. G. Nowak, Lattice points in large convex bodies, Monatsh. Math. 112 (1991), 61-72.

[11] C. G. Lekkerkerker, Geometry of Numbers, North-Holland, Amsterdam 1969.

[12] W. G. Nowak, On the lattice rest of a convex body in $\mathbb{R}^{s}$, Arch. Math. (Basel) 45 (1985), 284-288.

[13] - On the lattice rest of a convex body in $\mathbb{R}^{s}, I I$, ibid. 47 (1986), 232-237.

[14] -, On the lattice rest of a convex body in $\mathbb{R}^{s}$, III, Czechoslovak Math. J. 41 (116) (1991), 359-367.

[15] E. C. Titchmarsh, On Epstein's zeta-function, Proc. London Math. Soc. (2) 36 (1934), 485-500.

[16] - , The lattice points in a circle, ibid. 38 (1934), 96-115.

MATHEMATISCHE FAKULTÄT

FRIEDRICH-SCHILLER-UNIVERSITÄT

D-O-6900 JENA, GERMANY
INSTITUT FÜR MATHEMATIK UNIVERSITÄT FÜR BODENKULTUR GREGOR MENDEL-STRASSE 33 A-1180 WIEN, AUSTRIA 